\title{
Design and Implementation of Wireless Data Link Calibration Equipment of UAV
}

\begin{tabular}{|c|c|c|c|c|}
\hline LUO Jun & LU Wen-jun & YE Fei & SUN Shuang-xi & PENG Dong-hui \\
\hline New Star Research & New Star Research & New Star Research & Dongfeng Electrical & New Star Research \\
\hline $\begin{array}{l}\text { Institute of Applied } \\
\text { Technology }\end{array}$ & $\begin{array}{l}\text { Institute of Applied } \\
\text { Technology }\end{array}$ & $\begin{array}{l}\text { Institute of Applied } \\
\text { Technology }\end{array}$ & $\begin{array}{c}\text { Technology Co., Ltd. } \\
\text { Anhui }\end{array}$ & $\begin{array}{c}\text { Institute of Applied } \\
\text { Technology }\end{array}$ \\
\hline $\begin{array}{c}\text { Hefei, China } \\
\text { Loujun08@163.com }\end{array}$ & $\begin{array}{c}\text { Hefei, China } \\
\text { 1wj2963@sina.com }\end{array}$ & Hefei, China & Hefei, China & Hefei, China \\
\hline
\end{tabular}

\begin{abstract}
UAV system with a wireless data link for the calibration and test objects, designed and implemented a wireless data link system calibration equipment, medium-range UAV system to solve the simple means of technical support, equipment calibration testing process takes a long time, personnel, results poor reliability and accuracy problems. Test and results showed that the use of force: a reasonable system design, calibration, testing methods of science, advanced technology, stable performance, with medium-range UAV wireless data link for the key performance indicators calibration and testing functions.
\end{abstract}

Keywords-unmanned aerial vehicles, wireless data link, transmit power, receiver sensitivity, bit error rate

\section{INTRODUCTION}

UAV system, wireless data link system performance impact of its operational use is always a key factor in grassroots units not equipped with the appropriate testing equipment, only use low-power near-field signal for qualitative features observed; separated by aircraft and ground stations $5 \mathrm{~km}$ away from the high power signal pull test, the wireless data link to a rough assessment of the technical state of the system. Sometimes the signal is unstable and smooth and can not clear why. Through to the transmit power, receive sensitivity, bit error rate measurement parameters, comprehensive and accurate assessment of the UAV data link system wireless technology performance, provide the basis for the UAV operational commanders. Medium-range UAV system to the existing test methods based qualitative test, test indicator species, a small number of test results unreliable, difficult to accurately assess medium-range UAV system-wide performance. In the medium-range UAV wireless data link control loop calibration test in the test methods introduced in order to send remote commands and receive telemetry data, through two lines to send and receive, in the process of calibration and testing form a closed signal detection loop, build a dynamic online test environment, the state controlled UAV system calibration and testing of integrated performance. Detection method to solve a single system, low accuracy, quantitative analysis of the problem can not be.

Wireless data link system calibration equipment including power meters, programmable attenuation boxes, error analyzer, using a combination box structure.

\section{PROGRAMMABLE ATTENUATORS BOX}

Programmable attenuation box by the digitally controlled attenuator, power splitter, and semi-steel cable assembly, composed of a fixed attenuator, which digitally controlled attenuator for the active devices, easily influenced by ambient temperature is the power attenuation changes. Focus on the temperature of the module to do error correction, built-in temperature devices and temperature correction database. Other parts are passive devices, value stability, good pre-calibrated to ensure the system measurement requirements. From the splitter to divert part of the small-signal RF power meter, used for real-time monitoring of all the RF frequency band small-signal power for the system to accurately calculate the proper working of the receiver low.

\section{Wide Dynamic RANGe High ACCURACy Power METER}

Power meter in the wireless data link system, transmission equipment, the receiving device detection is an essential link. UAVs equipped for wireless data link system and the characteristics of RF signals, both to detect when the power meter to meet the high-power security, not to be burned, but also to ensure that when a small power range and accuracy. Be developed for the characteristics to meet the equipment requirements of the power meter indicator.

\section{A. Power Probe Design}

Films for thin-film thermocouple power sensor design, the need to ensure that the RF input matching condition; as thin-film thermocouple chip operating temperature range $0{ }^{\circ} \mathrm{C} \sim+45^{\circ} \mathrm{C}$ relatively wide, the use of insulation materials for the power sensor design, thermocouple inside the probe to ensure that power by the chip temperature and other environmental factors is small, the temperature coefficient of correction into the power meter within; Thermocouple voltage detected is relatively small $(0.01 \mathrm{mV})$, with a good stability low-noise amplifier, to obtain high sensitivity.

\section{B. Power indicator circuit design}

24-bit AD converter circuit of the indicator part of the design to achieve a less than $0.01 \mathrm{mV}$ voltage measurement, and data acquisition rate of 100 times $/ \mathrm{ms}$, to improve measurement efficiency; design frequency correction factor to improve the power meter to measure accurately sex.

\section{Calibration power meter calibration factor}

$\mathrm{C}$-band power in the power meter by the thermocouple probe and the corresponding indicator composed of two different ranges of power the probe and only a 
medium-power indicator. Its composition and physical

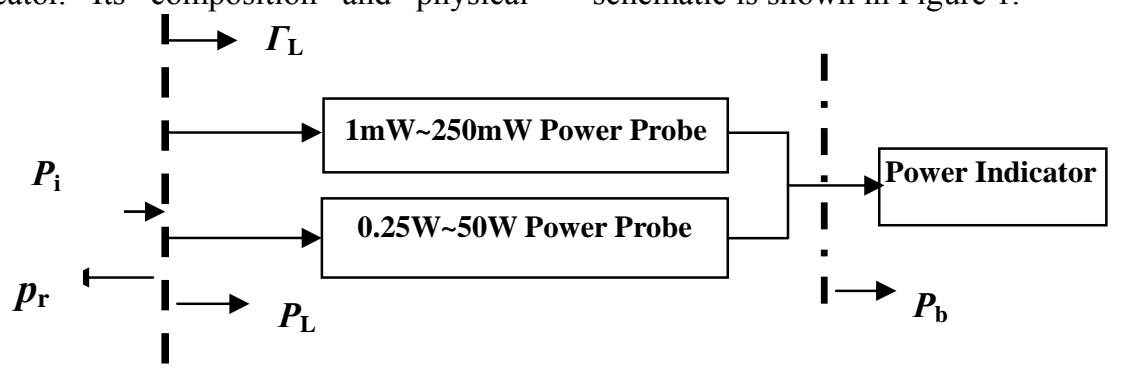

Fig.1 C-band power meter in composition theory

Developed in order to measure the power meter to measure the accuracy of the power needed for its calibration factor and calibration values to correct power meter readings. With a power meter to measure signal power $\mathrm{Pi}$, due to the power probe will usually generate the reflected power $\operatorname{Pr}$, which is the power reflection coefficient $\Gamma L$ the probe. To determine the input signal power $\mathrm{Pi}$, the introduction of the concept of calibration factors, and use of power meter calibration factor corrected instructions. Combined with the overall design, range of $1 \mathrm{~mW} \sim 250 \mathrm{~mW}$ and $250 \mathrm{~mW} \sim 50 \mathrm{~W}$ power range of power using alternative comparison method, respectively, low-power high-level standards and standards in the power of the power meter calibration factor for calibration, and scaling the value of write power calibration factor correction meter display program. After calibration, the power meter in the power measurement uncertainty of $3 \%$, higher than the design target.

\section{BERT}

Wireless transmission error rate is the UAV data link key performance indicators; wireless data link transmission channel has a special frame format. Currently only used for general-purpose BERT telecommunications and other areas of standard communication channel bit error rate test, the high reliability requirements of the channel, detect slow, can not be detected with a special frame format of dedicated channels, and expensive. Thus, according to UAV channel data frame format and error detection requirements, development of a particular frame can be adaptive channel format error detection analysis equipment as wireless data link detection system detects a critical error.

Common error rate detector for detecting a variety of standard in most high-speed channel, is not easy to detect wireless UAV data link system has a special frame format of dedicated communication channels, and expensive, to build complex test platform. Therefore, the UAV system error detection is developed by the error detector. Design of FPGA-based bit error rate tester program, using Alter Cyclone series FPGA (EP1C6240C) and related peripheral circuits to achieve error detection, error detector can work in a variety of modes, and error detection the results shown in the error detector directly on the LCD screen. UAV detection system calibration error detector host computer can implement asynchronous serial interface (UART) configuration error detector and read error information from the computer to complete a further error analysis.

System using FPGA as the core of the system, the control module, main module error detection, keyboard, LED / LCD display modules and other modules, the system achieved frame by frame option bytes can be timed single frame fixed bytes, optional time frames can be fixed bytes, an optional time frames can be fixed bytes and insert any intermediate PN code symbols such as the test channel performance.

UAV detection system based on the functional requirements for error detection, error detector working mode as follows:

To achieve a fixed length sequence of error detection code; To achieve within a certain time sequence of error detection; UAV data link uplink and downlink data frame format of the special error detection; UAV image transmission channel data frame format of the error detection.

Error detector schematic shown in Figure 2, FPGA internal logic of the code with the Verilog HDL hardware description language in Altera Quartus 6.0 integrated development environment development. 


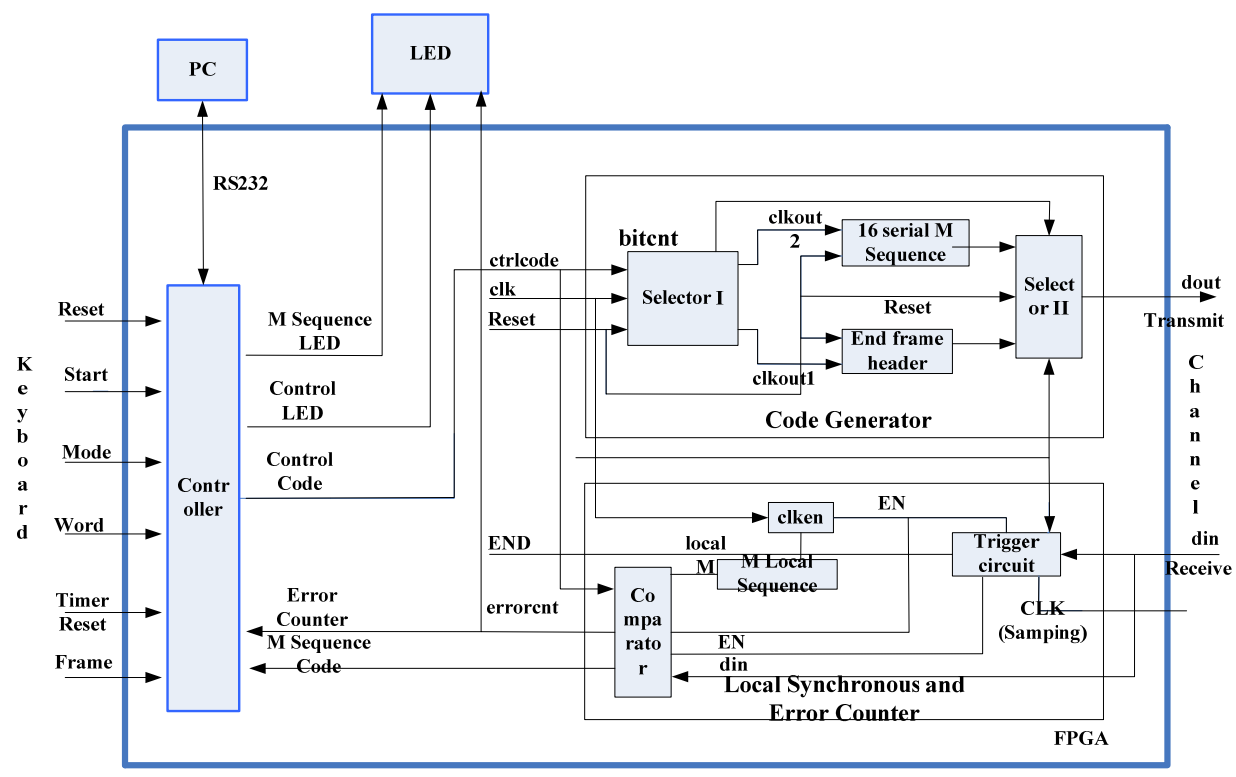

Fig.2 Block diagram of error detection

After testing, the error rate for the error detector $10^{-8}$, can be used for transfer rate lower than the $100 \mathrm{MHz}$ channel error detection, to meet the design requirements.

\section{REFERENCES}

[1] Li Xingshan, Zuo Yi. Automatic Test System Integration[M]. Beijing: Publishing House of Electronic Industry, 2004

[2] Wang Zhitian. Measurement of Radio Electronics[M], Beijing: Atomic Energy Press, 2002

[3] Wang Ting, Liu Ling, Po Shun Wang. Design of Control for HP8494/6H RF Attenuator[J]. Measurement and Control Technology, 2002, (10):61-63

[4] $\mathrm{Hu}$ Xiulin, Wu Xiangyang. Intellective Scheme for BER Testing Based on FPGA[J]. Measurement and Control Technology, 2006, (8):83-85

[5] Liu Jiang, Zhang Hongxiao, Liu Luokun. Design and realization of bit error ratio tester based on FPGA[J]. Micro Computer Information, 2005, (4):162-163

[6] Yu Zhenkun, Qu Wenying. Error Analysis on High Power RF Measurement[J]. Journal of Microwave, No. 3, 2006, (3):55-57 